\section{BMJ Open} Ophthalmology

\title{
Strengthening the integration of eye care into the health system: methodology for the development of the WHO package of eye care interventions
}

Stuart Keel (D) , ${ }^{1}$ Jennifer R Evans, ${ }^{2}$ Sandra Block, ${ }^{3}$ Rupert Bourne, ${ }^{4,5}$ Margarita Calonge, ${ }^{6}$ Ching-Yu Cheng, ${ }^{7,8}$ David S Friedman, ${ }^{9}$ João M Furtado, ${ }^{10}$ Rohit C Khanna, ${ }^{11}$ Wanjiku Mathenge, ${ }^{12}$ Silvio Mariotti, ${ }^{1}$ Elenoa Matoto, ${ }^{13}$ Andreas Müller, ${ }^{1} \mathrm{M}$ Mansur Rabiu, ${ }^{14}$ Tuwani Rasengane, ${ }^{15}$ Jialang Zhao, ${ }^{16}$ Richard Wormald, ${ }^{17,18}$ Alarcos Cieza ${ }^{1}$

\section{ABSTRACT}

Objective To describe the rational for, and the methods that will be employed to develop, the WHO package of eye care interventions (PECl).

Methods and analysis The development of the package will be conducted in four steps: (1) selection of eye conditions (for which interventions will be included in the package) based on epidemiological data on the causes of vision impairment and blindness, prevalence estimates of eye conditions and health facility data; (2) identification of interventions and related evidence for the selected eye conditions from clinical practice guidelines and high-quality systematic reviews by a technical working group; (3) expert agreement on the inclusion of eye care interventions in the package and the description of resources required for the provision of the selected interventions; and (4) peer review. The project will be led by the WHO Vision Programme in collaboration with Cochrane Eyes and Vision. A Technical Advisory Group, comprised of public health and clinical experts in the field, will provide technical input throughout all stages of development.

Results After considering the feedback of Technical Advisory Group members and reviewing-related evidence, a final list of eye conditions for which interventions will be included in the package has been collated.

Conclusion The PECl will support Ministries of Health in prioritising, planning, budgeting and integrating eye care interventions into health systems. It is anticipated that the $\mathrm{PECl}$ will be available for use in 2021 .

\section{INTRODUCTION}

Achieving Universal Health Coverage (UHC) is a WHO strategic priority. The objective of UHC is to ensure that all people have access to the promotive, preventive, curative and rehabilitative services they need, when and where they need them, without being exposed to financial hardship. ${ }^{1}$ In October 2019, the WHO launched the first World Report on Vision to emphasise the urgent

\section{Key messages}

What is already known about this subject? Eye care medicines and interventions are not integrated into the health insurance schemes in many low-income and middle-income countries.

\section{What are the new findings?}

- The package of eye care interventions will support countries to determine the (1) eye care interventions to prioritise, (2) workforce competencies, equipment medicines, consumables and assistive technologies necessary for the implementation of eye care interventions and (3) costs associated with the implementation of evidence-based eye care interventions.

How might these results change the focus of research or clinical practice?

It is the intention that the package of eye care interventions will take an important step to strengthening eye care within health systems, enabling more people to benefit from eye care interventions.

need for improved eye care globally, highlighting the role of eye care in contributing to UHC, and call for coordinated and concerted global action towards strengthening eye care in health systems. ${ }^{2}$

While many eye care interventions are cost-effective and feasible to implement, ${ }^{3}{ }^{4}$ persistent inequities in access to eye care services exist ${ }^{2}$. The gap between existing eye care needs and access to services is greater in low-income and middle-income countries and underserved populations, such as people living in rural areas, those with low incomes, women, older people, people with certain kinds of disability, indigenous populations and ethnic minorities ${ }^{2}$. Several factors accentuate the challenges associated with the delivery of equitable services. Major 


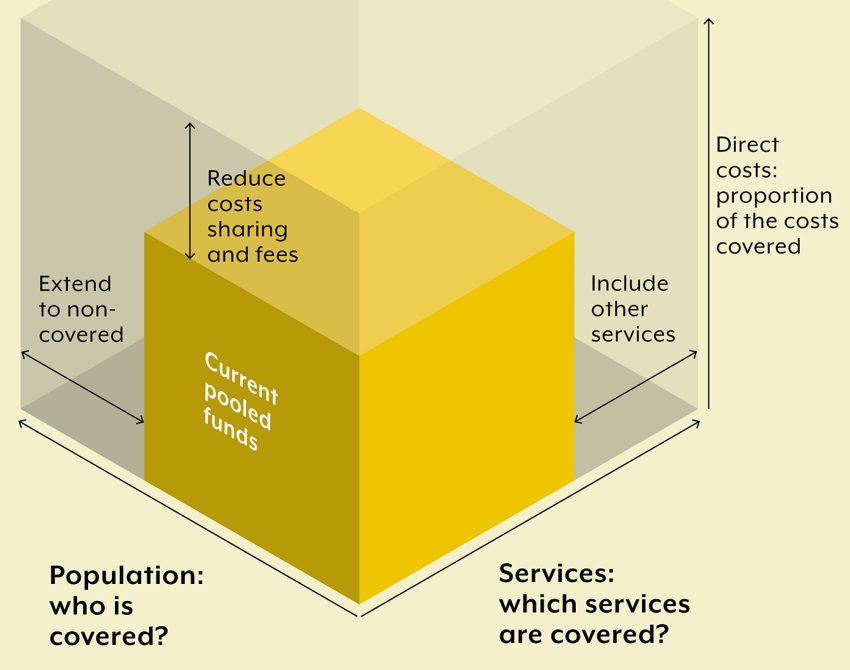

Figure 1 Dimensions of UHC. ${ }^{1}$ UHC, Universal Health Coverage.

demographic trends, including population ageing and lifestyle factors, will cause a substantial increase in the number of people with eye conditions ${ }^{5-7}$ and, thus, have a profound impact on the already strained health system and eye care workforce.

The costs associated with accessing eye care services pose a major barrier to addressing the inequities in access to, and provision of, eye care services across the population.
Therefore, an important component of UHC for eye care is that all people obtain the eye care services they need without risking financial hardship from unaffordable out-of-pocket payments. In order for countries to move towards UHC including eye care, they need to advance in three dimensions (figure 1$)^{1}$. First, priority eye care interventions and services included within health benefit packages need to be expanded; second, a greater proportion of the population needs to be covered; and third, out-of-pocket payments need to be reduced. Countries are required to make important choices when addressing these dimensions, such as which interventions and population groups should be prioritised.

To facilitate the choices that countries make when advancing UHC through eye care, WHO will develop a package of evidence-based eye care interventions to assist countries in making decisions on which interventions to prioritise, how these can be budgeted, and then integrated into national health services packages and policies. The objective of this paper is to describe the methods that will be employed to develop the package of eye care interventions (PECI).

\section{MATERIALS AND METHODS}

The development of the PECI will follow a stepwise approach with the involvement of different stakeholders (figure 2). WHO Vision Programme (WHO VP) will be responsible for the overall coordination of the project as well as technical and development work. Cochrane Eyes and Vision (CEV) will be an official partner in developing the PECI and will provide methods and evidence expertise. A WHO steering committee will oversee the project
Overall guidance: WHO Steering Group

The advisory board will be comprised of members of different WHO departments including WHO's Guidelines Review Committee Secretariat
Technical Advisory Group

The Technical Advisory Group will be comprised of public health and clinical experts in the field of eye care who will support the WHO Vision Programme along the different stages of development. 
and a Technical Advisory Group (TAG), comprised of public health, methods experts and clinical leaders in the field of eye care from all six WHO regions, will provide technical input throughout the different stages of development. Eye care experts will also collaborate with WHO in the identification of evidence on eye care interventions and creating descriptions of resources required for the provision of each intervention.

The declaration of interests of all external contributors will be assessed by an ethics officer from the Office of Compliance, Risk Management and Ethics at WHO. In the event that a conflict of interest (COI) exists, the ethics officer will advise on which COIs can be managed and which preclude participation.

\section{Phase 1. Selection of eye conditions}

The initial step of the development process involved the selection of eye conditions for which interventions will be included in the PECI. An initial selection performed by staff members from the WHO VP after considering the following evidence: (1) articles published in the scientific literature after 2010 reporting eye care facility data; (2) frequency data on the reasons for clinic attendance, over a predefined period, from selected health facilities located in a range of world regions and different resource settings; (3) global and/or regional population-based data on the causes of vision impairment and blindness among adult populations; (4) epidemiological data on the causes of childhood vision impairment and blindness; and (5) global and/or regional prevalence estimates of eye conditions.

TAG members were requested to independently review and provide written feedback on the collated data and preselected list of eye conditions, which was further discussed at the first TAG consultation of the PECI held on 25 March 2020.

\section{Phase 2. Identification of evidence on eye care interventions}

The identification of evidence on eye care interventions for each of the selected eye conditions will be performed by a Technical Working Group (TWG) made up of researchers from the field, including staff members from the WHO VP as well as members of external academic institutes.

Clinical Practice Guidelines (CPGs) will form the primary source of evidence for identifying relevant interventions to include in the PECI as they cover the continuum of care for a given eye condition by combining existing scientific evidence along with clinical expertise. Furthermore, CPGs cover the evidence gaps of the literature through expert consensus procedures. High-quality systematic reviews, such as Cochrane Systematic Reviews, will also be used to complement the evidence of CPGs when applicable (see Identification of systematic reviews section).

\section{Identification of CPGs}

The identification and selection of the CPGs will follow a stepwise approach (figure 3). An information specialist

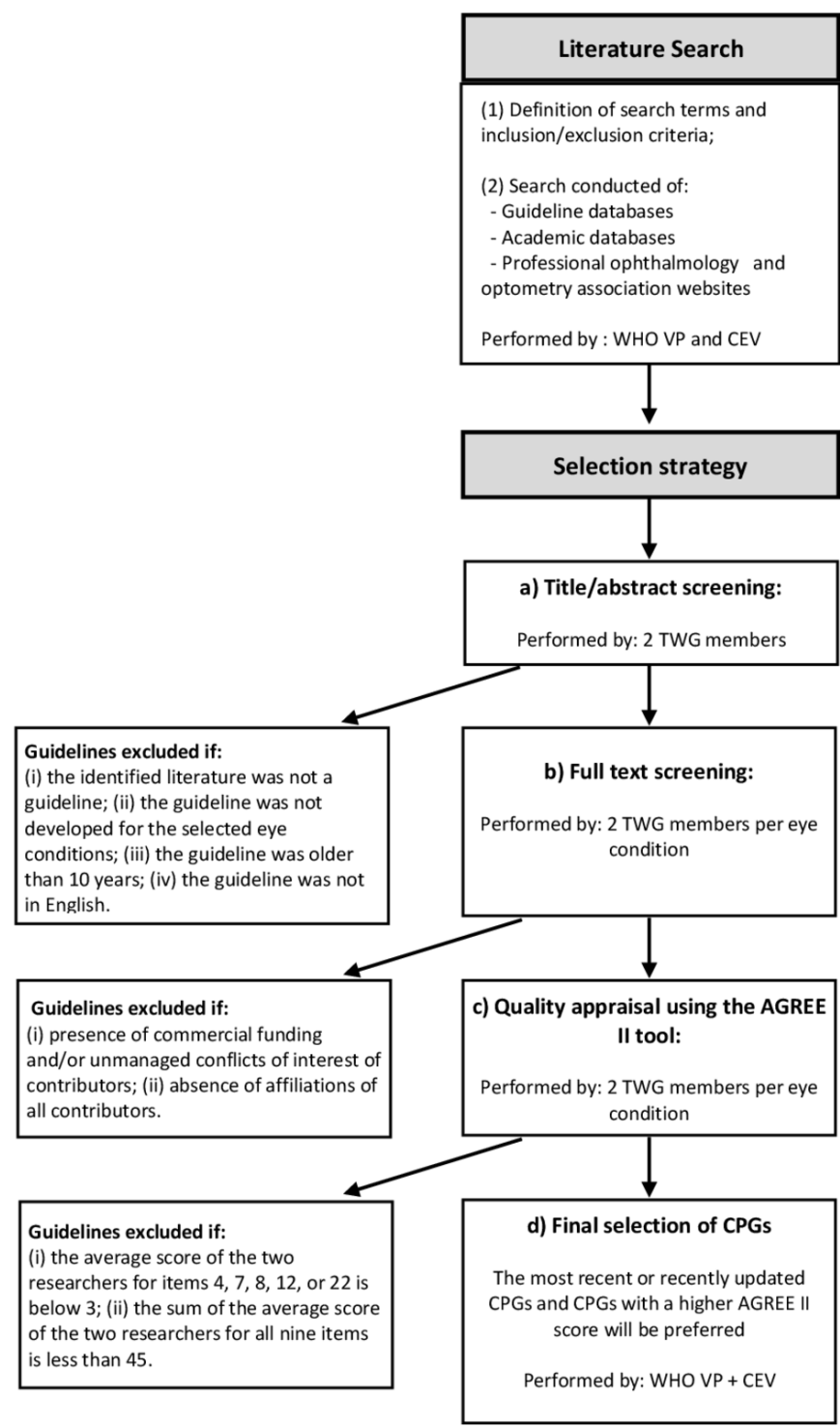

Figure 3 Flowchart for the identification of CPGs. CEV, Cochrane Eyes and Vision; CPGs, Clinical Practice Guidelines; TWG, Technical Working Group; WHO VP, WHO Vision Programme.

from CEV will design and carry out a single, systematic literature search of selected academic (MEDLINE, Embase, CINAHL, Global Health, Global Index Medicus) and guideline databases (online supplementary appendix 1). MeSH terms will be used where applicable. In addition, professional ophthalmology and optometry associations' websites will be searched for relevant guidelines (online supplementary appendix 1). The search terms and filters will be adapted according to the search options in the specific guideline databases and websites. All the searches will be limited to the last 10 years and to English language.

Two members of the TWG independently will screen the titles and abstracts of articles identified from the systematic literature searches. Abstrackr, a semiautomated online citation screening program, will be utilised where possible ${ }^{8}$. The following exclusion criteria will be 
applied: (1) the identified literature is not a guideline (ie, CPG or preferred practice pattern guideline); (2) the guideline is not related to the selected eye conditions; (3) the guideline is older than 10 years; and (4) the guideline is not in English. All disagreements will be resolved by discussion between a CEV representative and the WHO.

For each selected eye condition, two members of the TWG will independently conduct full-text screening of the guidelines identified as possibly relevant for the selected eye condition, adopting the following exclusion criteria: (1) presence of commercial funding and/or unmanaged conflicts of interest of contributors; and (2) absence of affiliations of all contributors. Disagreements will be resolved by discussion.

The quality of CPGs included after full-text screening will be evaluated by the same two members of the TWG using the 'Appraisal of Guidelines for Research and Evaluation' (AGREE II) tool $^{9}$ working independently. Nine items $(4,7,8,10,12,13,15,22$ and 23$)$ will be specifically used for the selection of guidelines. These items are the same as those utilised in the development of the package of rehabilitation interventions and were selected based on a consensus finding process among three researchers. ${ }^{10}$ If the rating of an item differs by more than two points between the two researchers, the results will be discussed between the two TWG members (and a representative of $\mathrm{WHO}$ or $\mathrm{CEV}$ if necessary) to reach consensus. If consensus cannot be reached, a third member of the TWG will be consulted. Following evaluation with the AGREE II tool, guidelines will be excluded if (1) the average score of the two researchers for items 4 , $7,8,12$, or 22 is below 3 ; and (2) the sum of the average score of the two researchers for all nine items is $<45$.

A maximum of five CPGs will be selected per eye condition. In the case that specific guidelines for children, youth and adults are available for a given eye condition, up to five CPGs will be included for each age group. Where more than five guidelines are identified for a given eye condition, the selection of CPGs will be made via consensus procedures among members of the WHO VP and CEV (with or without input from individual TAG members) after considering relevant information (eg, AGREE II score, date of publication, representativeness of the CPG (ie, country, regional, international scope)).

\section{Identification of systematic reviews}

Systematic reviews will be used to complement the evidence of CPGs when (1) no CPGs are identified for a given eye condition following screening and quality appraisal; (2) the selected high-quality CPGs provide contradictory recommendations for a given intervention; and/or (3) the CPG makes a recommendation on a given intervention based on evidence that is older than 10 years.

CEV will identify all Cochrane systematic reviews in the Cochrane library, published or updated in the last 10 years, either managed by CEV or with eyes and vision as a topic. Two members of the TWG will independently perform title and abstract screening. Systematic reviews will be excluded if they are not an intervention or diagnostic test accuracy review that is specifically related to the target eye condition. All disagreements will be resolved via discussion among TWG members with or without input from WHO and CEV representatives.

CEV US satellite maintains a database of other (ie, non-Cochrane) high-quality systematic reviews in eyes and vision ${ }^{11}$. Systematic reviews published in English within the last 10 years using the same exclusion criteria as that of Cochrane systematic reviews will be identified. In addition, to ensure only high-quality systematic reviews are selected, the two TWG members will independently appraise the quality of the methodology of each identified systematic review using the 'A MeaSurement Tool to Assess Systematic Reviews' 2 (AMSTAR 2) tool. The AMSTAR 2 expert group set out seven domain items (ie, items 2, 4, 7, 9, 11, 13 and 15) that may critically affect the validity of a review and its conclusions. ${ }^{12}$ A two-stage process will be followed: (1) reviews will be screened on item 2 (ie, whether the report of the review contained an explicit statement that the review methods were established prior to the conduct of the review); and (2) AMSTAR 2 assessment of the remaining critical domains will only be completed on reviews for which item 2 was rated as 'yes'. Disagreements will be resolved by discussion between the TWG members and CEV representative. Systematic reviews will only be selected if, following an adjudication process (where applicable), all of the remaining critical domain items $(4,7,9,11,13$ and 15) are rated as 'yes' or 'partial yes' by both researchers.

\section{Data extraction and preparation}

Data extraction will be completed by one member of the TWG and checked by another. Data extraction from CPGs will include information on the following domains: (1) information on the guideline (ie, title, authors, year of publication); (2) reference to the recommendations, interventions and related outcomes; (3) content and strength of the recommendation/s; and (4) quality of evidence related to the recommendation/s. For systematic reviews, information on the population, intervention and control, setting, statistical values for each of the analysis, number of studies and sample characteristics, summary of main results, certainty of evidence and adverse events will be extracted.

\section{Phase 3. Approval of evidence-based eye care interventions} and description of resources required for their provision Specific Development Groups (DGs) will be founded for each eye care subspecialty areas (eg, retina, anterior segment, glaucoma, paediatric, etc) and will include clinical, academic and public health professionals from all WHO regions (with a focus on low-income and middleincome countries), with working or research experience in the provision of interventions along the life course (paediatric and adult care). 
A three-step process will be carried out among each subspecialty-specific DG in order to (1) achieve consensus on the inclusion of evidence-based eye care interventions in the PECI for each eye condition; (2) identify any relevant interventions that were not included within the list formulated by the TWG to be considered for inclusion within the PECI (if applicable); and (3) agree on the service delivery platforms, workforce competencies and time and resources required to provide each of the selected interventions. This will consist of an initial online survey among each DG member, followed by data preparation by WHO (with or without input from specific DG members) and a web-based group discussion/s among DG members.

\section{Online preparatory survey}

Prior to undertaking the online survey, each DG member will receive the extracted information that was generated by the TWG, including full texts of the selected CPGs and the data extracted from the CPGs and systematic reviews (where applicable). For each given intervention, the DG members will be required to confirm whether they recommend the intervention for inclusion in the PECI based on the strength and quality of the recommendations, as well as its applicability and appropriateness in their own settings. If the intervention is recommended for inclusion in the PECI, the DG member will then (1) assign each intervention to one or more service delivery platforms (eg, school, outreach, hospital (in-patient or outpatient), clinic, home); and (2) identify missing interventions that were not identified by the TWG but are considered by the DG member as relevant to the eye condition.

\section{Data preparation}

Following the completion of the online survey by all members of the subspecialty DG, the WHO VP will collate a list of interventions in which majority agreement was achieved during the online survey and a separate list of interventions that did not achieve majority consensus, and thus require further deliberation. In addition, systematic literature searches will be coordinated with CEV for any missing interventions that were identified by the DG. Whenever the evidence supports the suggested assessment or intervention, it will be added to the list of interventions that requires further deliberation during the web-based group discussion.

For each of the interventions that achieved majority consensus during the online survey, WHO will be responsible for drafting descriptions of the required resources and other relevant information for the intervention including the (1) target population (stage of life course, special context target population); (2) workforce requirement; (3) most appropriate service delivery platform/s (based on the survey results); (4) time required to provide each activity/procedure related to the intervention (per unit/event); and (5) required health products including equipment, consumables and assistive products.

\section{Web-based group discussions}

The DG results from the online survey, coupled with additional data collated by WHO in the data preparation stage, will be presented to DG members prior to a web-based group discussions. The objectives of the initial group discussions are twofold. First, to confirm the list of interventions to be included in the package. This will involve achieving consensus on (1) interventions that did not achieve majority agreement for inclusion in the package during the online survey and (2) identified missing interventions after considering the evidence derived from the systematic literature searches. Second, to review and update (if applicable) data collated by $\mathrm{WHO}$ on the service delivery platforms, equipment and workforce requirements for interventions that achieved consensus during the online survey. Following this, WHO will collate data relating to service delivery platforms, equipment and workforce requirements for interventions identified in (1) and (2) above that achieved majority agreement for inclusion in the PECI and follow-up webbased group discussion/s will be held to review and update this information.

Prior to peer review and to avoid overlap in the provision of resources, discussions will be held between WHO and selected members from each DG to identify crosscutting interventions that for a multitude of reasons, including complementary workforce competencies and health product requirements, are relevant to the diagnosis, management or treatment of multiple eye conditions.

\section{Phase 4. Peer review}

A Peer Review Group (PRG) comprised of internal (WHO) members and eye care experts from all WHO regions will be formed. PRG members will be responsible for independently reviewing the package and providing feedback and recommendations for revision. The presented package will include a list of interventions with core data. All feedback and recommendations will be considered before producing the first version of the PECI.

\section{The patient and public involvement}

It was not deemed appropriate for patients and/or the public to be involved in the design, conduct, reporting or dissemination of this work.

\section{RESULTS}

\section{Selection of eye conditions for inclusion in the PECI}

After considering the feedback of TAG members and reviewing related evidence, a final list of eye conditions for inclusion in the PECI was collated (box 1).

\section{DISCUSSION}

This paper presents the methodology for the development of the WHO evidence-based PECI. In brief, the PECI will support countries to determine the (1) eye care interventions to prioritise, (2) workforce 
Box 1 Selection of eye conditions for inclusion in the PECI (not in order of priority)
1. Cataract
2. Congenital cataract
3. Refractive error
4. Diabetic retinopathy
5. Glaucoma
6. Congenital glaucoma
7. Age-related macular degeneration
8. Myopic macular degeneration
9. Amblyopia
10. Strabismus
11. Retinopathy of prematurity
12. Uveitis
13. Ocular trauma ${ }^{1}$
14. Ocular surface disorders ${ }^{2}$
a. Conjunctivitis (infective and allergic)
b. Dry eye disease
c. Keratoconus
d. Keratitis and corneal ulcer
e. Pterygium

15. Disorders of the eyelid and lacrimal system ${ }^{2}$

a. Ptosis, entropion and ectropion

b. Congenital anomalies

c. Blepharitis

d. Chalazion and hordeolum

${ }^{1}$ Interventions for the treatment of corneal opacities due to 'ocular trauma' and complications of 'ocular surface disorders' to be included. ${ }^{2}$ Key conditions have been defined under the broader categories of 'ocular surface disorders' and 'disorders of the eyelid and lacrimal system' in recognition of the different treatment approaches required and to facilitate the identification of evidence on interventions for each condition. Eye conditions 1-13 will be all inclusive in terms of their coverage of interventions for the variety of subclassifications. $\mathrm{PECl}$, package of eye care interventions.

competencies, equipment, medicines, consumables and assistive technologies necessary for the implementation of eye care interventions and (3) costs associated with the implementation of evidence-based eye care interventions. It is anticipated that the PECI will be available for use in 2021 .

Eye care medicines and interventions are not integrated into the health insurance schemes in many low-income and middle-income countries ${ }^{4}$. An important component of UHC for eye care is that all people obtain the eye care services they need without risking financial hardship from unaffordable out-ofpocket payments. Thus, in order to promote equitable access to services, including protection against financial hardship, a shift is required to ensure that high-priority eye care interventions are included in service packages covered by prepaid pooled financing. This is particularly important for the poor. Once finalised, the PECI will be integrated in the WHO's UHC compendium of interventions, containing interventions across all of WHO's programmatic health areas, and linked with the WHO OneHealth Tool, a single framework for planning, costing, impact analysis, budgeting and financing. When considered together with reliable data about the population eye care needs, these resources will facilitate discussions in lowincome and middle-income countries around what eye care services to provide within their national health services packages.

While the primary audience for the PECI will be Ministries of Health $(\mathrm{MoH})$ and other ministries involved in eye care service delivery in low-income and middle-income settings, there are a number of other potential end-users of the PECI. Government service providers will be able to use the PECI to plan and implement specific eye care interventions in their service programmes. Training facilities can use the PECI to develop their curricula for the training of health workers involved in eye care. In addition, based on the results of stage 2 of the development processthe systematic identification and quality appraisal of CPGs and systematic reviews-researchers will be able to identify current gaps in evidence and define research strategies to address these gaps.

The scope of eye conditions included in the PECI goes beyond the leading causes of vision impairment and blindness. The inclusion of eye conditions that may not typically cause vision impairment, such as dry eye disease, conjunctivitis, pterygium and blepharitis, is important for several reasons. First, these conditions are troublesome and painful and often severely impact on quality of life. ${ }^{13-15}$ Second, these conditions are frequently among the leading reasons for presentation to eye care services in all countries and thus expose individuals to financial burden. ${ }^{16-20}$ The list of eye conditions included in the priority PECI is not meant to be exhaustive or exclusive, but rather represent a selection of conditions and eye care interventions that are supported by high-quality evidence and that are applicable to most low and intermediate resource settings. Future work will involve refining and expanding the PECI, drawing on newly available evidence and newly collected data on service provision. It is important to note that intervention packages for vision rehabilitation, trachoma, onchocerciasis, vitamin A deficiency, measles and rubella were not considered in the PECI as they are encompassed under the WHO Department of Neglected Tropical Diseases and WHO's Programmes on Nutrition and Rehabilitation, respectively. Once finalised, the selected interventions will undergo a process of prioritisation, with input from clinical and public health specialists from all WHO regions, in order to develop recommendations for a 'basic' package of interventions to assist countries when making choices of which interventions to prioritise in their service packages.

Some potential limitations should be considered regarding the methodology employed for the development of the PECI. First, the utilisation of CPGs as the primary source of evidence for the selection of 
interventions may be viewed as controversial given that the vast majority of scientific evidence forming the basis of the CPGs often originates from highincome settings, and therefore may not be pertinent to low resource settings. To address this, eye care experts from low-income and middle-income settings will be involved in all stages of development of the package, including during the process of defining the workforce competencies and resource requirements for the delivery of interventions. Second, limiting the search of CPGs and systematic reviews to the English language (only) will result in relevant CPGs published in other languages being overlooked. This decision was one of feasibility given the labour-intensive nature of screening CPGs published in all official WHO languages and later translation of the extracted information. In instances where there is an absence of evidence, WHO representatives will consult specific TAG members to determine benefit and feasibility of identifying evidence from CPGs published in other languages. Lastly, using the eye condition as a starting point for the PECI development may be perceived as counterintuitive for the integration of eye care interventions within the broader health system. As mentioned previously, the PECI will be integrated in the WHO's UHC compendium of interventions, containing interventions across all of WHO's programmatic health areas. In addition, the WHO VP will work closely with representatives from other related WHO programmes, including ageing, primary care, diabetes and maternal and child health, to ensure relevant PECI are aligned and integrated within the care packages of these programmes.

To address many of the challenges facing the eye care sector, including those relating to changing demographics, inequities in access, and lack of integration-eye care needs to be an integral part of UHC. In summary, the development of the PECI described herein aims to move forward the agenda of eye care as part of UHC by providing countries with information on evidence-based eye care interventions, including resource requirements for their implementation, to facilitate the integration of eye care into national health services packages and policies. It is therefore the intention that the PECI will take an important step to strengthening eye care within health systems, enabling more people to benefit from eye care interventions.

\section{Author affiliations}

${ }^{1}$ Department of Noncommunicable Diseases, World Health Organization, Geneva, Switzerland

${ }^{2}$ International Centre for Eye Health, London School of Hygiene and Tropical Medicine, London, United Kingdom

${ }^{3}$ Illinois College of Optometry, Chicago, United States

${ }^{4}$ Cambridge University Hospitals, Cambridge, United Kingdom

${ }^{5}$ Vision \& Eye Research Institute, School of Medicine, Anglia Ruskin University, Cambridge, United Kingdom
${ }^{6}$ Institute of Applied OphthalmoBiology, University of Valladolid and CIBER-BBN (Biomedical Research Networking Center Bioengineering, Biomaterials and Nanomedicine), Carlos III National Institute of Health, Valladolid, Spain ${ }^{7}$ Ophthalmology \& Visual Sciences Academic Clinical Program, Duke-NUS Medical School, Singapore

${ }^{8}$ Singapore Eye Research Institute, Singapore National Eye Centre, Singapore ${ }^{9}$ Massachusetts Eye and Ear, Harvard University, Boston, United States

${ }^{10}$ Division of Ophthalmology, Ribeirão Preto Medical School, University of São Paulo, Ribeirão Preto, Brazil

${ }^{11}$ Allen Foster Community Eye Health Research Centre, Gullapalli Pratibha Rao International Centre for Advancement of Rural Eye care, L V Prasad Eye Institute, Hyderabad, India

${ }^{12}$ Rwanda International Institute of Ophthalmology, Kigali, Rwanda

${ }^{13}$ Colonial War Memorial Hospital, Suva, Fiji

${ }^{14}$ Noor Dubai Foundation, Dubai Health Authority, Dubai, United Arab Emirates

${ }^{15}$ Department of Optometry, University of the Free State and Universitas Hospital,

Bloemfontein, South Africa

${ }^{16}$ Department of Ophthalmology, Peking Union Medical College Hospital, Eye Research Center Chinese Academy of Medical Sciences, Peking Union Medical College, Beijing, China

${ }^{17}$ NIHR Biomedical Research Centre, Moorfields Eye Hospital NHS Foundation Trust, London, United Kingdom

${ }^{18} \mathrm{UCL}$ Institute of Ophthalmology, London, United Kingdom

Correction notice This article has been corrected since it was published. The acknowledgement and contributors statements have been updated.

Acknowledgements We would like to thank Dr Susan Norris from the WHO Guideline Review Committee Secretariat for her valuable support in the development of the protocol.

Contributors $S K$ and $A C$ were involved in planning and drafting the manuscript. JRE, SB, RB, MC, CY-C, DF, JMF, RCK, WM, SM, EM, AM, MR, TR, JZ and RW reviewed and revised the draft. All authors read and approved the final manuscript. The views expressed in this paper are those of the authors and do not necessarily reflect the views of WHO.

Funding The authors have not declared a specific grant for this research from any funding agency in the public, commercial or not-for-profit sectors.

Competing interests None declared.

Patient consent for publication Not required.

Provenance and peer review Not commissioned; externally peer reviewed.

Data availability statement All data relevant to the study are included in the article.

Open access This is an open access article distributed in accordance with the Creative Commons Attribution Non Commercial (CC BY-NC 4.0) license, which permits others to distribute, remix, adapt, build upon this work non-commercially, and license their derivative works on different terms, provided the original work is properly cited, appropriate credit is given, any changes made indicated, and the use is non-commercial. See: http://creativecommons.org/licenses/by-nc/4.0/.

ORCID iD

Stuart Keel http://orcid.org/0000-0001-6756-348X

\section{REFERENCES}

1 WHO. Strategizing National health in the 21st century: a Handbook. WHO, 2016.

2 WHO. World report on vision. Geneva: World Health Organization, 2019.

3 Horton S, Gelband H, Jamison D, et al. Ranking 93 health interventions for low- and middle-income countries by costeffectiveness. PLoS One 2017;12:e0182951.

4 Tahhan N, Papas E, Fricke TR, et al. Utility and uncorrected refractive error. Ophthalmology 2013;120:1736-44.

5 Holden BA, Fricke TR, Wilson DA, et al. Global prevalence of myopia and high myopia and temporal trends from 2000 through 2050. Ophthalmology 2016;123:1036-42.

6 Tham Y-C, Li X, Wong TY, et al. Global prevalence of glaucoma and projections of glaucoma burden through 2040: a systematic review and meta-analysis. Ophthalmology 2014;121:2081-90. 
7 Wong WL, Su X, Li X, et al. Global prevalence of age-related macular degeneration and disease burden projection for 2020 and 2040: a systematic review and meta-analysis. Lancet Glob Health 2014;2:e106-16.

8 Wallace B, Small K, Brodley C, et al. Deploying an interactive machine learning system in an evidence-based practice center: abstrackr. Proceedings of the 2nd ACM SIGHIT international health informatics symposium 2012:819-24.

9 Brouwers MC, Kerkvliet K, Spithoff K, et al. The agree reporting checklist: a tool to improve reporting of clinical practice guidelines. BMJ 2016;352:i1152.

10 Rauch A, Negrini S, Cieza A. Toward strengthening rehabilitation in health systems: methods used to develop a who package of rehabilitation interventions. Arch Phys Med Rehabil 2019;100:2205-11.

11 Mayo-Wilson E, Ng SM, Chuck RS, et al. The quality of systematic reviews about interventions for refractive error can be improved: a review of systematic reviews. BMC Ophthalmol 2017;17:164.

12 Shea BJ, Reeves BC, Wells G, et al. AMSTAR 2: a critical appraisal tool for systematic reviews that include randomised or nonrandomised studies of healthcare interventions, or both. BMJ 2017;358:j4008.

13 Barber L, Khodai O, Croley T, et al. Dry eye symptoms and impact on vision-related function across international Task force guidelines severity levels in the United States. BMC Ophthalmol 2018;18:260.

14 Miljanović B, Dana R, Sullivan DA, et al. Impact of dry eye syndrome on vision-related quality of life. Am J Ophthalmol 2007;143:409-15.

15 Pouyeh B, Viteri E, Feuer W, et al. Impact of ocular surface symptoms on quality of life in a United States Veterans Affairs population. Am J Ophthalmol 2012;153:1061-6.

16 Adio AO, Alikor A, Awoyesuku E. Survey of pediatric ophthalmic diagnoses in a teaching hospital in Nigeria. Niger $\mathrm{J}$ Med 2011;20:105-8.

17 Biswas J, Saha I, Das D, et al. Ocular morbidity among children at a tertiary eye care hospital in Kolkata, West Bengal. Indian J Public Health 2012;56:293-6.

18 Eballe AO, Bella LA, Owono D, et al. [Eye disease in children aged 6 to 15 years: a hospital-based study in Yaounde]. Sante 2009;19:61-6.

19 Hassan MB, Olowookere SA, Adeleke NA, et al. Patterns of presentations at a free eye clinic in an urban state Hospital. Niger $J$ Clin Pract 2013:16:145-8.

20 Mehari ZA. Pattern of childhood ocular morbidity in rural eye Hospital, central Ethiopia. BMC Ophthalmol 2014;14:50. 